\title{
Hunger Modulates the Responses to Gustatory Stimuli of Single Neurons in the Caudolateral Orbitofrontal Cortex of the Macaque Monkey
}

Edmund T. Rolls, Zenon J. Sienkiewicz, and Simon Yaxley

University of Oxford, Department of Experimental Psychology, South Parks Road, Oxford OX1 3UD, UK

Key words: taste, hunger, satiety, gustatory cortex, orbitofrontal cortex, primate, sensory-specific satiety

Abstract

1. In order to determine whether the responsiveness of neurons in the caudolateral orbitofrontal cortex (a secondary cortical gustatory area) is influenced by hunger, the activity evoked by prototypical taste stimuli (glucose, $\mathrm{NaCl}, \mathrm{HCl}$, and quinine hydrochloride) and fruit juice was recorded in single neurons in this cortical area before, while, and after cynomolgous macaque monkeys were fed to satiety with glucose or fruit juice.

2. It was found that the responses of the neurons to the taste of the glucose decreased to zero while the monkey ate it to satiety during the course of which his behaviour turned from avid acceptance to active rejection.

3. This modulation of responsiveness of the gustatory responses of the neurons to satiety was not due to peripheral adaptation in the gustatory system or to altered efficacy of gustatory stimulation after satiety was reached, because modulation of neuronal responsiveness by satiety was not seen at earlier stages of the gustatory system, including the nucleus of the solitary tract, the frontal opercular taste cortex, and the insular taste cortex.

4. The decreases in the responsiveness of the neurons were relatively specific to the food with which the monkey had been fed to satiety. For example, in seven experiments in which the monkey was fed glucose solution, neuronal responsiveness decreased to the taste of the glucose but not to the taste of blackcurrant juice. Conversely, in two experiments in which the monkey was fed to satiety with fruit juice, the responses of the neurons decreased to fruit juice but not to glucose.

5. These and earlier findings lead to a proposed neurophysiological mechanism for sensory-specific satiety in which the information coded by single neurons in the gustatory system becomes more specific through the processing stages consisting of the nucleus of the solitary tract, the taste thalamus, and the frontal opercular and insular taste primary taste cortices, until neuronal responses become relatively specific for the food tasted in the caudolateral orbitofrontal cortex (secondary) taste area. Then sensory-specific satiety occurs because in this caudolateral orbitofrontal cortex taste area (but not earlier in the taste system) it is a property of the synapses that repeated stimulation results in a decreased neuronal response.

6. Evidence was obtained that gustatory processing involved in thirst also becomes interfaced to motivation in the caudolateral orbitofrontal cortex taste projection area, in that neuronal responses here to water were decreased to zero while water was drunk until satiety was produced.

\section{Introduction}

In order to analyse the neural control of feeding, the activity of single neurons is being recorded during feeding in brain regions implicated in feeding in the monkey (Rolls, 1981, 1986, 1987; Rolls and Rolls, 1982). It has been found that a population of neurons in the lateral hypothalamus and adjoining substantia innominata of the monkey respond to the sight and/or taste of food (Rolls et al., 1976). Part of the evidence that these neurons are involved in the control of the responses which are made to food when hungry is that these neurons only respond to food when the monkey is hungry (Burton et al., 1976; Rolls et al., 1985). Indeed, it has been suggested that the modulation of the sensory response to a motivationally relevant sensory stimulus such as the taste of food by motivational state, for example hunger, is one important way in which motivational behaviour is controlled (Rolls, 1975, 1982).

We have recently shown that this modulation of taste-evoked input by motivation is not a property found in early stages of the primate gustatory system. Single neuron activity in the nucleus of the solitary tract, the rostral half of which receives first-order gustatory afferents in the monkey (Scott et al., 1985) and the

Correspondence to: E. T. Rolls, as above.

Received 25 February 1988, revised 15 July 1988, accepted 18 July 1988 


\section{Taste in macaque monkey}

caudal half of which receives afferents from the viscera, is not attenuated by feeding to satiety (Yaxley et al., 1985). Thus taste processing at this early stage of the taste system does not appear to be modulated by satiety, the signals for which include gastric distension (Gibbs et al., 1981) as well as other signals (Rolls and Rolls, 1977).

Anatomical studies have shown that the next projection in the taste system in the primate is from the nucleus of the solitary tract directly to the parvicellular part of the ventral posteromedial thalamic nucleus (Beckstead and Norgren, 1979; Beckstead et al., 1980; Norgren, 1984). This thalamic region then projects to the frontal opercular taste cortex and to the insular taste cortex (Pritchard et al., 1986), which in turn both project to a caudolateral area of the orbitofrontal cortex (Wiggins et al., 1988). In the frontal opercular taste cortex (Scott et al., 1986b) and in the insular taste cortex (Yaxley et al., 1989), the responses of neurons to gustatory stimuli are more specifically tuned to gustatory stimuli than are neurons in the nucleus of the solitary tract (Scott et al., 1986a), but in neither the frontal opercular taste cortex (Rolls et al., 1988) nor the insular taste cortex (Yaxley et al., 1988) does hunger modulate the responsiveness of single neurons to gustatory stimuli. In the caudolateral orbitofrontal cortex gustatory area neuronal responses are more sharply tuned to gustatory stimuli than in earlier parts of the taste system (Rolls, Sienkiewicz, and Yaxley, in preparation). It was the purpose of the experiments described here to investigate whether hunger modulates the responsiveness of single neurons in this cortical region to taste stimuli.

To ensure that the results were relevant to the normal control of feeding (and were not due for example to abnormally high levels of artificially administered putative satiety signals such as gastric distension or plasma glucose), we allowed the monkeys to feed until they were satiated, and determined whether this normal and physiological induction of satiety influenced the responsiveness of neurons in the gustatory cortex, which were recorded throughout the feeding, until satiety was reached. The recordings were made in the monkey in order to make the results as relevant as possible to our understanding of sensory processing and the control of feeding, and its disorders, in the human.

\section{Methods}

The methods used were similar to those described previously (Rolls et al., 1988; Rolls et al., 1976; Burton et al., 1976; Rolls et al., 1977; Sanghera et al., 1979; Scott et al., 1986a, b; Yaxley et al., 1985), and are presented here as briefly as possible, except where they differ.

\section{Recording}

Two male cynomolgus monkeys, Macaca fascicularis, weighing $3.8-4.0 \mathrm{~kg}$ were implanted under anaesthesia with sodium thiopentone ( $5 \%$; initial dose $1 \mathrm{ml}$ i.v., preceded by tranquillization with ketamine at $10 \mathrm{mg} / \mathrm{kg}$ i.m.) with stainless-steel holders on which a Kopf adaptor could be fitted during recording sessions. After 1 or 2 weeks, daily recording sessions were initiated. Neuronal activity was recorded using glass-coated tungsten microelectrodes (after Merrill and Ainsworth, 1972), while the monkey sat in a primate chair with head restraint to provide recording stability. The electrode was protected by a guide tube which ended just below the lignocaine-anaesthetized dura. The signal from the microelectrode was passed through a field effect transistor source follower amplifier mounted on the microdrive, amplified by conventional band-pass filtered amplifiers, and displayed on an oscilloscope. The monkey was fed, and given water ad lib, at the end of each daily recording session, so that he was approximately $18 \mathrm{~h}$ food-and water-deprived during the recording sessions.

\section{Stimuli and stimulus delivery}

Five stimuli plus water were applied to the tongue during a recording session. Four were prototypes of the four basic tastes, each at a concentration determined from intensity-response functions in these same subjects (Scott et al., 1986b). These were 1.0 $\mathrm{M}$ glucose, $1.0 \mathrm{M} \mathrm{NaCl}, 0.01 \mathrm{M} \mathrm{HCl}$, and $0.001 \mathrm{M}$ quinine hydrochloride. The fifth stimulus was $20 \%$ blackcurrant juice (Ribena, Beecham Products, Brentford, UK). This was chosen as a stimulus which, because of its palatability and complexity of taste, would be readily accepted and which would activate many gustatory neurons.

The stimuli were delivered through a hand-held syringe in quantities of approximately $0.5 \mathrm{ml}$. Manual delivery was used to ensure replicable gustatory stimulation of a large and nearly constant receptive field throughout a recording session despite different mouth and tongue positions adopted by the monkeys as the palatability of the solutions varied with the stimulus quality and level of satiety.

Neurons in the caudolateral orbitofrontal cortex taste area responded with quite sharp tuning to the four prototypical taste stimuli, and did not respond to somatosensory stimulation (see Rolls et al., in preparation). The stimuli to which different neurons responded best included glucose, blackcurrant juice, water, and $\mathrm{NaCl}$, with $\mathrm{HCl}$ and quinine hydrochloride being relatively ineffective gustatory stimuli for the majority of the neurons analyzed (Rolls et al., in preparation).

\section{Requirements for conducting a satiety experiment}

Twelve satiety experiments were performed at different sites within the caudolateral orbitofrontal cortex gustatory area. Each was separated from the others by at least 2 days so as to permit the effects of repletion to dissipate. In order to initiate a satiety experiment, three conditions had to be satisfied.

1. Gustatory responsiveness to the satiating chemical. The neuronal response elicited by application of the stimulus which would subsequently be used to satiate the monkey had to be robust. Since satiety was induced in most cases by glucose and in some cases by fruit juice, most of the neurons studied in the satiety experiments responded well to sweet stimuli. In one additional experiment, the effects of drinking water to satiety on the responses of a neuron which responded strongly to water were investigated.

2. Recording stability. After a neuron had been found in the gustatory cortex, the stability of the recording and of the evoked neuronal response were tested periodically over the next 30-60 min before a decision was made to begin the experiment.

3. Avidness for the satiating chemical. A series of objective criteria for the avidness of acceptance has been developed (Rolls et al., 1977; Rolls et al., 1988). A satiety experiment was not initiated unless a monkey's behaviour warranted a rating of at least +1.0 on a scale of +2.0 (acceptance) to -2.0 (rejection) (see below). In practice this required an efficient search for a neuron in the gustatory cortex and the achievement of a stable 
recording with a minimum of stimulus presentations, so that the monkey was still hungry when the experiment started.

\section{Criteria for acceptance or rejection}

Scores on the scale of acceptance or rejection were based on the following behavioural criteria:

+2.0: maximal acceptance: reaching for the solution with hands and mouth; avid licking.

+1.0: clear acceptance: opening the mouth, licking and swallowing the solution.

0.0: neutrality: swallowing the solution when placed in the mouth; absence of avidness; no attempt made to obtain the solution.

-1.0 : clear rejection: pursing the lips to prevent administration of the solution; failure to swallow all of the solution placed in the mouth.

-2.0: maximum rejection: pursing the lips and closing the teeth; using the tongue to eject delivered solution; swallowing little; using the hands to push away the solution.

If the behaviour was intermediate between these types, then intermediate scores were given.

\section{Protocol}

If the criteria for conducting a satiety experiment were satisfied, the following protocol was invoked.

1. The gustatory neuronal response to each of the five sapid stimuli plus water was determined by application of $0.5 \mathrm{ml}$ of each solution. Each application was followed by a $1.0 \mathrm{ml}$ water rinse, and a minimum period of $30 \mathrm{sec}$ of rest. The stimulus series was then repeated. The total testing time was approximately 12 min, and the volume consumed was a maximum of $16 \mathrm{ml}$.

2. The monkey's acceptance-rejection score for the satiating solution was determined by observing his response as $0.5 \mathrm{ml}$ was applied to the tongue.

3. The monkey was fed a $50 \mathrm{ml}$ aliquot of the satiating solution. In nine cases this was $20 \% \mathrm{w} / \mathrm{v}$ glucose. This was the primary agent for inducing satiety because, insofar as postabsorptive processes are involved, these will be expedited by glucose which does not need to be metabolized before absorption. In one case $20 \%$ blackcurrant juice was used, so as to provide a wider range of information on satiety, and in one case pure apple juice was used, also to test the effects of satiety with a substance other than glucose, and because the neuron responded well to this solution. In one case (for a neuron which responded to water), water was used in the satiety experiment. All satiating solutions were delivered by a syringe. The duration of administration was approximately two minutes for the initial aliquot, and as much as four minutes for the last.

4. The monkey's acceptance-rejection score to the satiating solution was reassessed.

5. Steps 1 to 4 were repeated through as many cycles as were required to attain a behavioural score of -1.5 to -2.0 . This typically involved five $50 \mathrm{ml}$ aliquots over a period of $60 \mathrm{~min}$. Conventional satiety, defined by the stage at which the subject would stop working to obtain food, would normally correspond to a rating of 0.0 to -0.5 . Thus the feeding used in these experiments was sufficient to produce normal, self-determined, behavioural satiety, to ensure that if there was a modulation of the neuronal responses by satiety, the degree of satiety induced in the experiments was sufficient for the modulation to be manifested. Further evidence that normal satiety was induced in these experiments was the large quantity of glucose ingested by the monkeys. The quantity was at least $150 \mathrm{ml}$ of $20 \%$ glucose solution (as shown in Fig. 1), that is, at least $30 \mathrm{~g}$ of glucose for a $4 \mathrm{~kg}$ monkey. After satiety was reached, and feeding had stopped, multiple further measurements were taken of the neuronal responses to each of the sapid solutions and to water.

\section{Analysis}

The responses of single neurons were acquired, analysed, and displayed on-line by a PDP-11 computer. Mean discharge rates were computed during either control periods or stimulus presentation, with the analysis extending $5 \mathrm{sec}$ from stimulus onset. Neuronal activity, together with stimulus markers, was also recorded on magnetic tape for subsequent analysis, which included the calculation and display of peristimulus time histograms in $\mathbf{5 0}$ ms bins.

\section{Localization of recording sites}

The position of each recording site was determined in two ways. First, following each track, X-ray photographs were taken from frontal and lateral perspectives (cf. Aggleton and Passingham, 1981). Recording sites could then be reconstructed to within 250 $\mu \mathrm{m}$ by reference to deep electrodes permanently implanted at planes close to those of the recording track. The positions of the deep electrodes were subsequently determined histologically. Second, in the final several tracks, microlesions were made through the recording electrode ( $60 \mu \mathrm{A}$ for $60 \mathrm{sec}$, electrode negative). After the final experiment, tranquilization with ketamine (10 $\mathrm{mg} / \mathrm{kg}$ i.m.) was followed by a lethal intravenous dose of sodium pentobarbitone $(30 \mathrm{mg} / \mathrm{kg}$ initially). Perfusion was with $0.9 \%$ saline followed by formal-saline. The brains were placed in sucrose formalin for at least 7 days, after which $50 \mu \mathrm{m}$ serial frozen sections were cut and stained with cresyl violet and by the Gallyas (1979) method for myelin (for which $25 \mu$ sections were used).

\section{Results}

The effects of feeding the monkey to satiety on the responsiveness of neurons recorded in the caudolateral orbitofrontal cortex are shown in Figure 1. Each part of the figure shows one experiment in which the effect of satiety on the responsiveness of a single neuron to gustatory stimuli was measured. The responses to the satiating chemical and the spontaneous firing rate of the neuron, are shown at the different stages of each experiment. In nine of the experiments shown in Figure 1 the satiating chemical was glucose, in one blackcurrant juice, in one apple juice, and in one water, as labelled. It is clear that in all cases satiety produced a major reduction in the responsiveness of the neurons to the food, and that by the time the monkey had fed enough to be fully satiated (acceptance indices of -1 to -2 ), the neurons had ceased or almost ceased to respond to the food. In all nine cases in which satiety was produced by glucose, in the case in which it was produced by blackcurrant juice, and in the case in which it was produced by apple juice, there was a significant reduction $(\mathrm{p}<$ 0.01 ) in the response of the neuron to the food produced by feeding to satiety, and when satiety was complete, there was little or no difference between the firing rate of the neuron when the food was being tasted and the spontaneous firing rate (see Fig.1). 

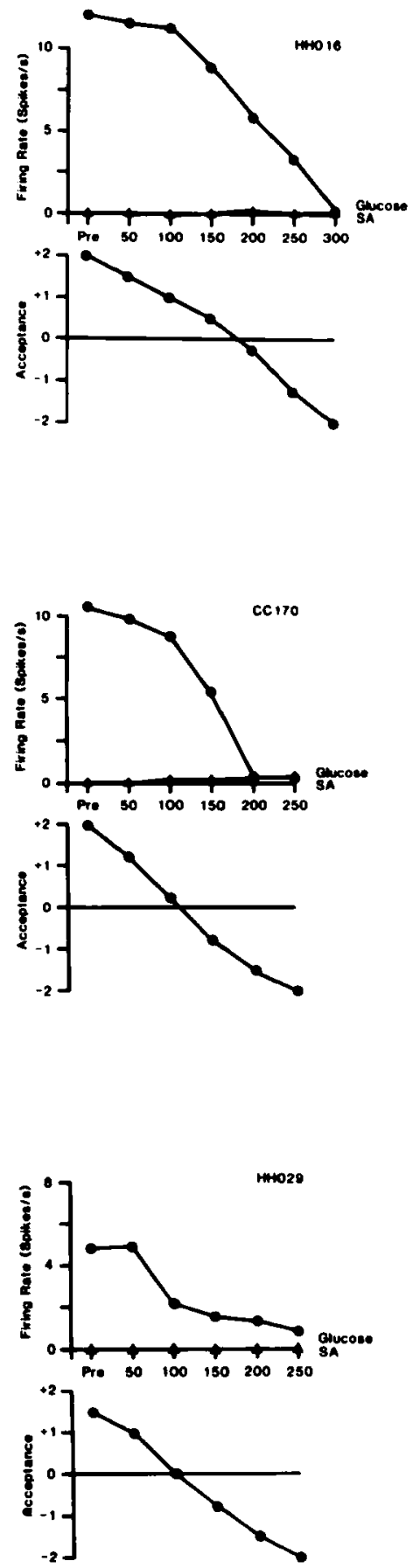
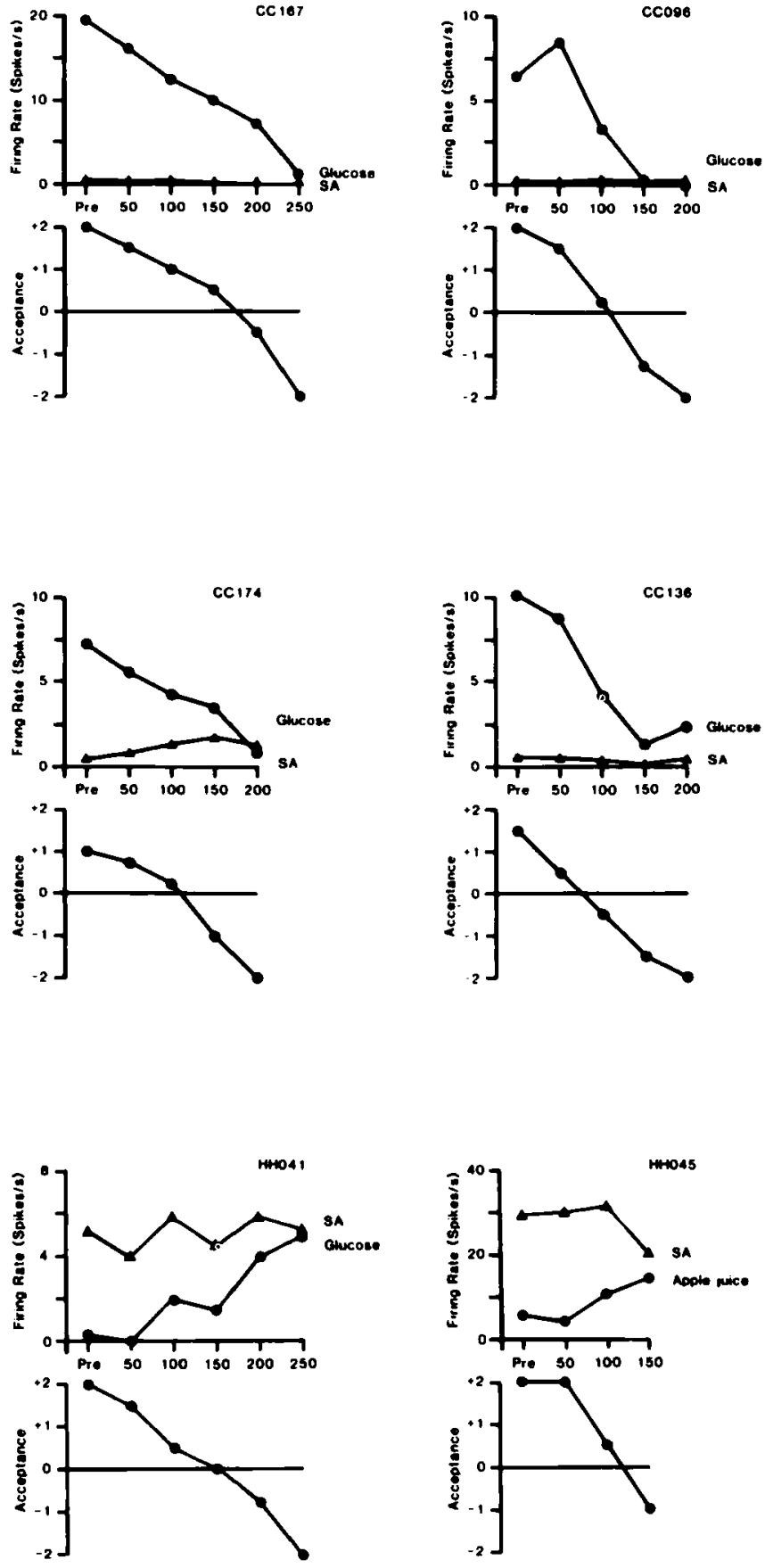
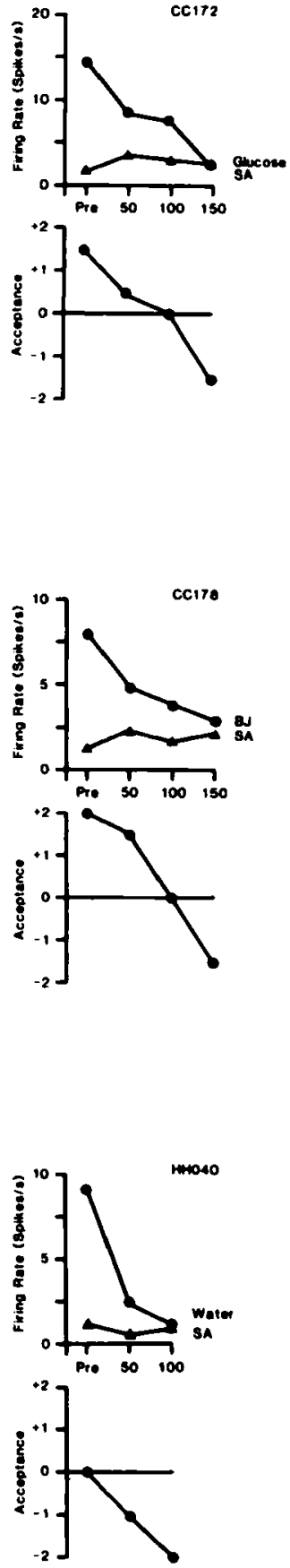

VOLUME INGESTED (ML)

Fig. 1. Effects of feeding to satiety on the neural response (spikes/s) to the solution on which the monkey was satiated, for 11 separate experimental runs in which the neuron responded to sweet stimuli, and one in which the neuron responded to water. The spontaneous firing rate is also indicated (SA). Below the neuronal response data for each experiment, the behavioural measure of the acceptance or rejection of the solution on a scale from +2 to -2 (see text) is shown. The solution used to feed to satiety was $20 \%$ glucose, except for neuron CCl 78 , for which blackcurrant juice was used; neuron $\mathrm{HH} 045$ for which apple juice was used; and neuron HH040 for which water was used. The monkey was fed $50 \mathrm{ml}$ of the solution at each stage of the experiment as indicated along the abscissa, until he was satiated as shown by whether he accepted or rejected the solution. Pre-the firing rate of the neuron before the satiety experiment started. 


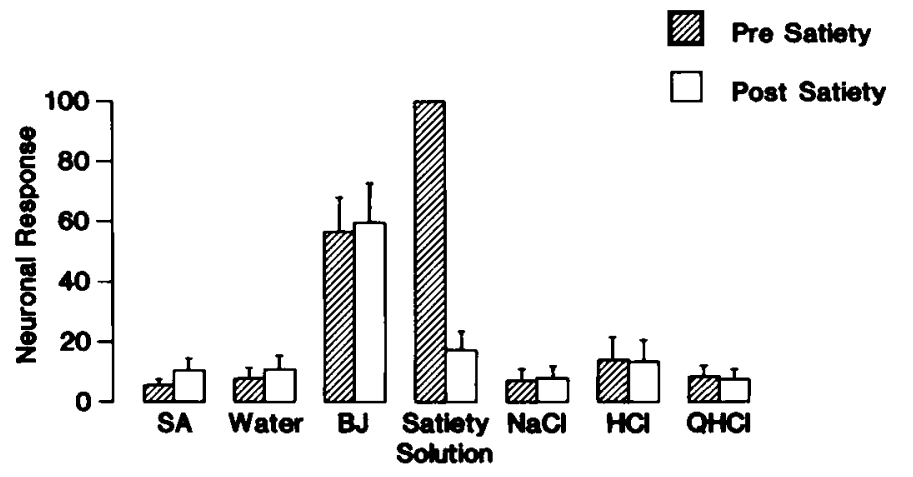

Fig. 2. The neuronal response before and after satiety to each of the gustatory stimuli, and to the solution on which the monkey was satiated. The results are expressed relative to the response before satiety obtained to the solution on which the monkey was satiated (set at $100 \%$ ), and the data are averaged over the 10 experimental runs shown in Figure 1 in which the satiating solution was glucose or blackcurrant juice, with the mean and sem shown.

It will be noticed that some of the neurons in the caudolateral orbitofrontal cortex responded by a decrease of firing rate (e.g. neurons HH041 and HH045 in Fig. 1), and that for these neurons also the response of the neuron to the tastant, that is its change of firing rate when the tastant was present from the spontaneous firing rate, also decreased towards zero during satiation. It is also clear from Figure 1 that satiety typically produced very little alteration in the spontaneous firing rate of the neurons.

The results of feeding the monkey to satiety on the neuronal responses to each of the gustatory stimuli are shown in Figure 2. The means and standard errors of the firing rates of the neurons before the satiety test was started, that is when the monkey was approximately $18 \mathrm{~h}$ food-deprived, and after the monkey had been fed to satiety, are indicated. The results are shown averaged over the 11 experiments in which neurons responded to sweet stimuli in order to provide a summary of the effect of satiety on processing in the clOFC. It is clear that there was a major decrease in the response of the neurons to the taste of the solution with which the monkey was satiated $(t=5.54, \mathrm{df}=10, \mathrm{p}<0.001)$, and no significant change in the gustatory responses to any other of the gustatory stimuli used.

Another fascinating finding of these experiments is evident in Figure 2. Whereas the neuronal response to the solution with which the monkey was fed was abolished by satiety, the neuronal response to another sweet solution, blackcurrant juice, was unaltered. There thus is some specificity of the effects of the satiety, which is sensory-specific in that it occurs to the sweet solution with which the monkey was satiated, but not to another sweet solution. This effect is shown in more detail (with the standard errors of the neuronal responses) for two neurons in Figure 3 . The effect was not due to inherent greater palatability of the blackcurrant juice, in that when blackcurrant juice was drunk to satiety in one experiment illustrated in Figure 4, the neuron ceased responding to blackcurrant juice, but still responded to glucose. The same effect was found in the experiment in which apple juice was ingested to produce satiety.

There are also neurons in this region which respond when water is tasted by the monkey (Rolls et al., 1988b). To determine whether thirst modulates the responses of these neurons to the taste of

\section{OFC}
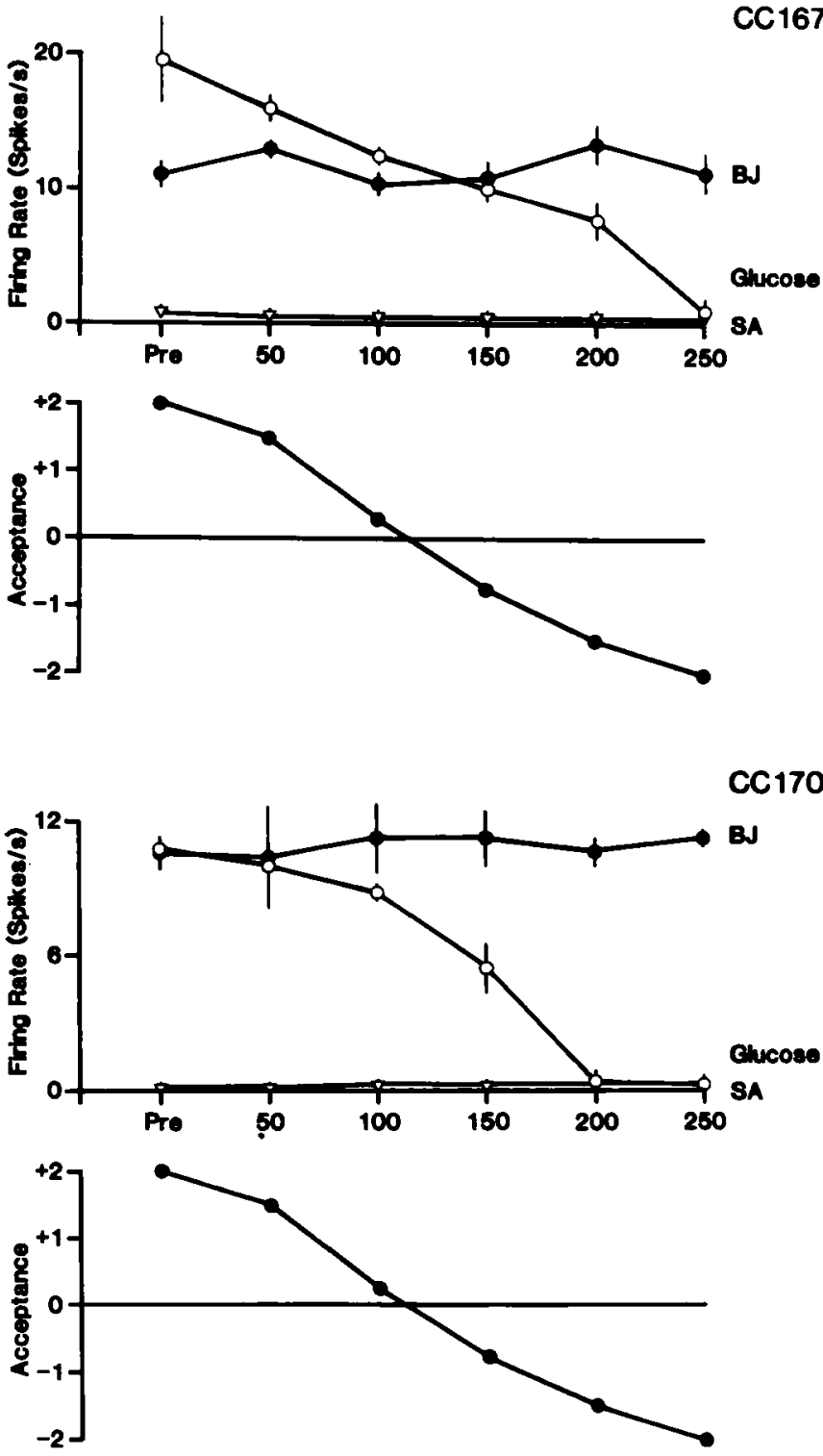

Fig. 3. The effect of feeding to satiety with glucose solution on the responses of two neurons to the taste of glucose and of blackcurrant juice. The conventions are as in Figure 1. The bars represent the standard errors of the means.

water, the $18 \mathrm{~h}$ water-deprived monkey was allowed to drink to satiety. It was found that the neuronal response to the taste of the water was decreased by drinking water to satiety (see neuron HH040 in the bottom right of Fig. 1).

The sites of the neurons on which these satiety experiments were performed are shown in Figure 5. All the neurons were in the caudolateral orbitofrontal cortex taste area described by Rolls et al. (in preparation).

\section{Discussion}

These results show that in the taste area in the caudolateral orbitofrontal cortex of the primate, satiety decreases the responses 

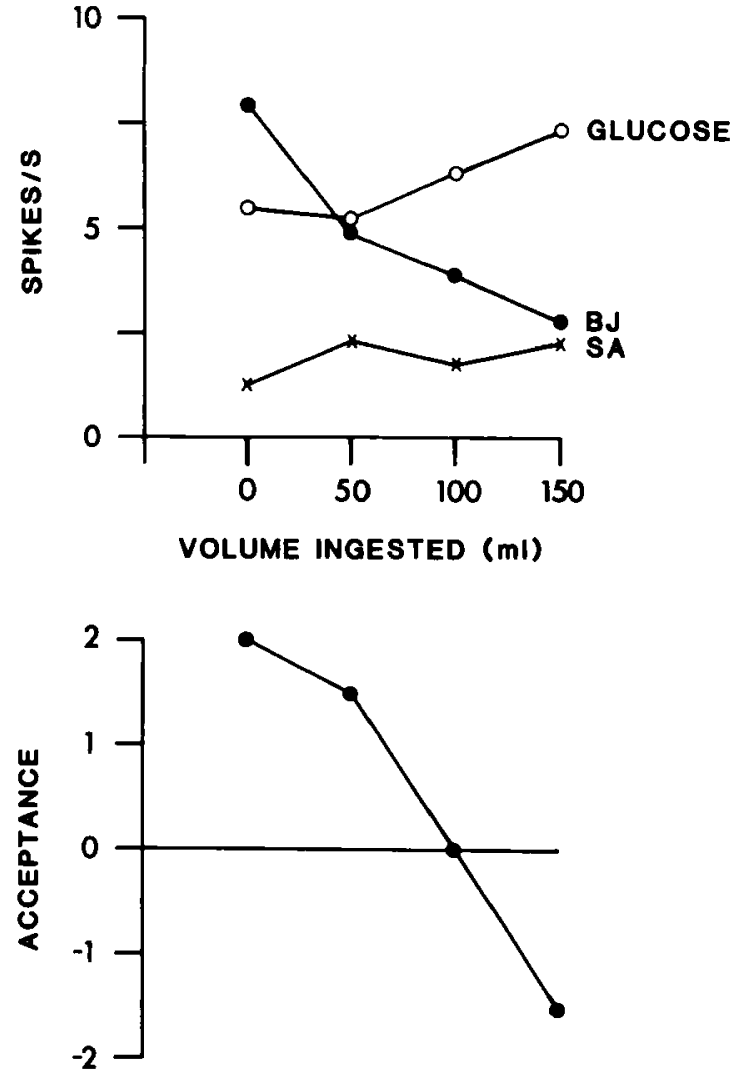

FIG. 4. The effect of feeding to satiety with blackcurrant juice solution on the responses of a neuron to the taste of glucose and of blackcurrant juice. The conventions are as in Figure 1. The bars represent the standard errors of the means.

of neurons to the taste of the food with which the monkey is satiated. This is in contrast to gustatory neuronal responses in the nucleus of the solitary tract (Yaxley et al., 1985), and in the two cortical gustatory areas which project into the caudolateral orbitofrontal cortex (Wiggins et al., 1988), the frontal opercular taste cortex (Scott et al., 1986b) and the insular taste cortex (Yaxley et al., 1988a), in which feeding to satiety does not modulate the responsiveness of the neurons (Rolls et al., 1988a; Yaxley et al., 1988b).

This evidence shows that the reduced acceptance of food which occurs when food is eaten to satiety, and the reduction in the pleasantness of its taste (Cabanac, 1971; Rolls, 1986; Rolls et al., 1981a, b, 1982; Rolls and Rolls, 1977, 1982; Rolls et al., 1983), are not produced by a reduction in the responses of neurons in the nucleus of the solitary tract or frontal opercular or insular gustatory cortices to gustatory stimuli. Indeed, after feeding to satiety, humans reported that the taste of the food on which they had been satiated tasted almost as intense as when they were hungry, though much less pleasant (Rolls et al., 1983). This comparison is consistent with the possibility that activity in the frontal opercular and insular taste cortices as well as the nucleus of the solitary tract does not reflect the pleasantness of the taste of a food, but rather its sensory qualities independently of motivational state. On the other hand, the responses of the neurons in the caudolateral orbitofrontal cortex taste area (as shown here) and in the lateral hypothalamus (Rolls et al., 1986)

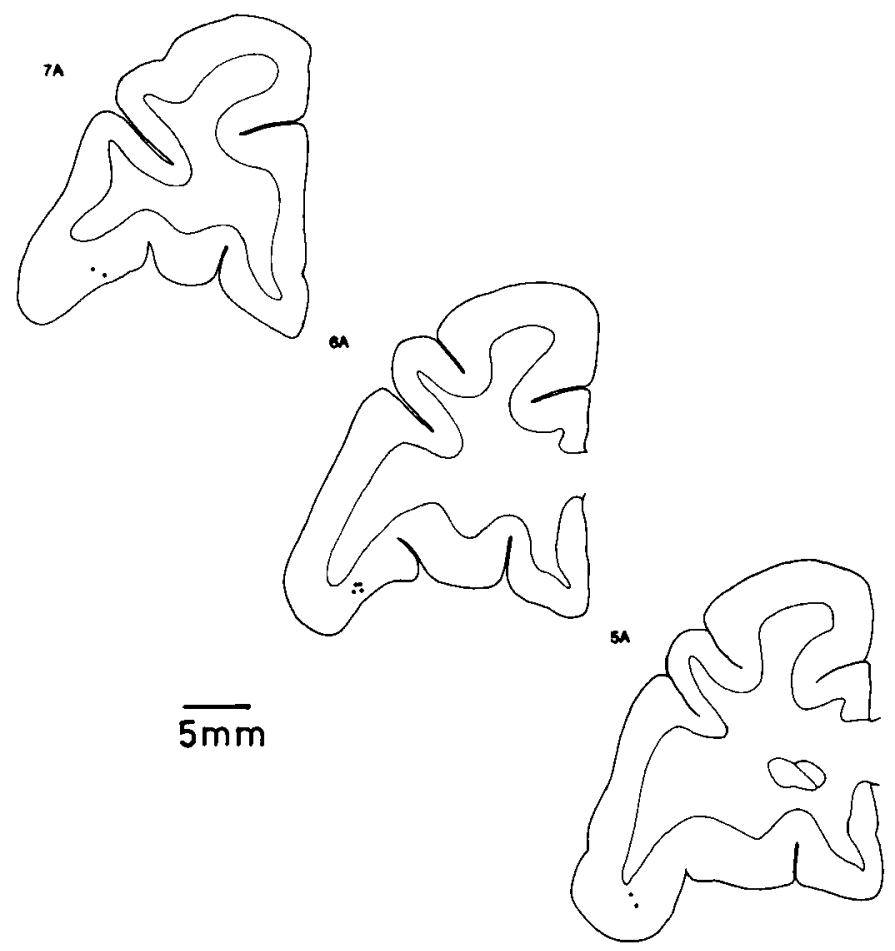

FIG. 5. Recording sites in the caudolateral orbitofrontal cortex at which the satiety experiments were performed. The numbers refer to the number of $\mathrm{mm}$ the coronal sections shown are anterior to the posterior wing of the sphenoid (see Aggleton and Passingham, 1981).

are modulated by satiety, and it is presumably in areas such as these that neuronal activity may be related to whether a food tastes pleasant, and to whether the food should be eaten.

The present results also provide evidence on the nature of the mechanisms which underlie sensory-specific satiety. Sensoryspecific satiety, as noted above, is the phenomenon in which the decrease in the palatability and acceptability of a food which has been eaten to satiety are partly specific to the particular food which has been eaten (Rolls, 1986; Rolls et al., 1981a, b, 1982; Rolls and Rolls, 1977, 1982; Rolls et al., 1983). The results just described suggest that such sensory-specific satiety cannot be largely accounted for by adaptation at the receptor level, in the nucleus of the solitary tract, or in the frontal opercular or insular gustatory cortices, to the food which has been eaten to satiety, otherwise modulation of neuronal responsiveness should have been apparent in the recordings made in these regions. Indeed, the findings suggest that sensory-specific satiety is not represented in the primary gustatory cortex. Instead, neuronal activity in the caudolateral orbitofrontal cortex is very closely related to sensoryspecific satiety, as shown here.

These findings lead to a proposed neuronal mechanism for sensory-specific satiety. The tuning of neurons becomes more specific for gustatory stimuli through the nucleus of the solitary tract, gustatory thalamus, frontal opercular taste cortex, and insular taste cortex (see e.g. Rolls, 1987, 1988; Rolls et al., in preparation). Satiety, habitation, and adaptation are not features of the responses in these regions. The tuning of neurons becomes even more specific in the caudolateral orbitofrontal cortex, but here habituation with a time course of several minutes and which 
lasts for $1-2 \mathrm{~h}$ is a feature of the synapses which are activated, and in addition there may be some effect of satiety by internal signals such as gastric distension and glucose utilization. Because of the relative specificity of the tuning of orbitofrontal taste neurons, this results in a decrease in the response to that food, but different foods continue to activate other neurons. One output of these neurons may be to the hypothalamic neurons with foodrelated responses, for their responses to the taste of food show a decrease which is partly specific to a food which has just been eaten to satiety (Rolls et al., 1986).

The theoretical significance of this computational architecture is probably as follows. If satiety were to operate at an early level of sensory analysis, then because of the broadness of tuning of neurons, responses to non-foods would become attenuated as well as responses to foods (and this could well be dangerous if poisonous non-foods became undetectable). This argument becomes even more compelling when it is realized that satiety typically shows some specificity for the particular food eaten, with others not eaten in the meal remaining relatively pleasant (see above and Rolls et al., 1984). Unless tuning were relatively fine, this mechanism could not operate, for reduction in neuronal firing after one food had been eaten would inevitably reduce behavioural responsiveness to other foods. Indeed, it is of interest to note that such a sensory-specific satiety mechanism can be built by arranging for tuning to particular foods to become relatively specific at one level of the nervous system (as a result of categorization processing in earlier stages), and then at this stage (but not at prior stages) to allow habituation to be a property of the synapses, as proposed above.

It is useful to compare these findings in the primate with the data obtained in the rat. There is evidence that in the rat gastric distension and increases in blood glucose level can decrease neural responses in the gustatory part of the nucleus of the solitary tract (Giza and Scott, 1983; Glenn and Erickson, 1976). There are a number of possible reasons why different results were obtained in the rat from those obtained in the monkey. First, the results from the monkey were obtained from an alert animal feeding to normal self-determined satiety, whereas those from the rat reflect responsiveness under artificial conditions. For example, the blood glucose levels in the rat, which exceeded $200 \mathrm{mg} \%$ (Giza and Scott, 1983), may have been higher than those which occur normally. Second, the rat studies were performed under anaesthesia. Third, it is possible that the control systems for feeding operate differently in the rat and the primate. It does at least appear that the anatomy of the gustatory system is different in the rat and the monkey, as described above. Thus in the rat, it is possible that with the close interconnection of the nucleus of the solitary tract and the hypothalamic and forebrain areas implicated in motivational control, hedonic-motivational coding may be apparent far peripherally and may not be well distinguished from quality-intensity coding, whereas in the monkey, there may be more distinction. Part of the evolutionary reason why there might be such a difference in gustatory information processing in the rat and the monkey is that cross-modal associations (for example matching the sight of a particular object such as an orange in a tree with the taste of that object) may be particularly well developed in primates. However, it may be noted that even in the rat, peripheral modulation of gustatory responsiveness by satiety is unlikely to be able to account for satiety to a taste stimulus, in that the modulation may only decrease the neuronal response to the gustatory stimulus by perhaps 30\% (Giza and Scott, 1983; T. R. Scott, personal communication, 1985).

\section{References}

Aggleton, J. P., Burton, M. J., and Passingham, R. E. (1980) Cortical and subcortical afferents to the amygdala of the rhesus monkey (Macaca mulatta). Brain Res. 190: 347-368.

Aggleton, J. P. and Passingham, R. E. (1981) Stereotaxic surgery under $\mathrm{X}$-ray guidance in the Rhesus monkey, with special reference to the amygdala. Exp. Brain Res. 44: 271-276.

Bagshaw, M. H. and Pribram, K. H. (1953) Cortical organization in gustation (Macaca mulatta). J. Neurophysiol. 16: 499-508.

Beckstead, R. M. and Norgren, R. (1979) An autoradiographic examination of the central distribution of the trigeminal, facial, glossopharyngeal, and vagal nerves in the monkey. J. Comp. Neurol. 184: $455-472$.

Beckstead, R. M., Morse, J. R., and Norgren, R. (1980) The nucleus of the solitary tract in the monkey: projections to the thalamus and brainstem nuclei. J. Comp. Neurol. 190: 259-282.

Benjamin, R. M. and Burton, H. (1968) Projection of taste nerve afferents to anterior opercular-insular cortex in squirrel monkey (Saimiri sciureus). Brain Res. 7: 221-231.

Burton, M. J., Rolls, E. T., and Mora, F. (1976) Effects of hunger on the responses of neurones in the lateral hypothalamus to the sight and taste of food. Exp. Neurol. 51: 668-677.

Cabanac, M. (1971) Physiological role of pleasure. Science 173: 11031107.

Gallyas, F. (1979) Silver staining of myelin by means of physical development. Neurol. Res. 1: 203-209.

Gibbs, J., Maddison, S. P., and Rolls, E. T. (1981) Satiety role of the small intestine examined in sham-feeding rhesus monkeys. J. Comp. Physiol. Psychol. 95: 1003-1015.

Giza, B. K. and Scott, T. R. (1983) Blood glucose selectively affects tasteevoked activity in rat nucleus tractus solitarius. Physiol. Behav. 31: 643650.

Glenn, J. F. and Erickson, R. P. (1976) Gastric modulation of gustatory afferent activity. Physiol. Behav. 16: 561-568.

Merrill, E. G. and Ainsworth, A. (1972) Glass-coated platinum-plated tungsten microelectrodes. Med. Biol. Eng., 10: 662-672.

Mesulam, M.-M. and Mufson, E. J. (1982a) Insula of the old world monkey. I. Architectonics in the insulo-orbito-temporal component of the paralimbic brain. J. Comp. Neurol. 212: 1-22.

Mesulam, M.-M. and Mufson, E. J. (1982b) Insula of the old world monkey. III. Efferent cortical output and comments on function. J. Comp. Neurol. 212: 38-52.

Mufson, E. J., Mesulam, M.-M., and Pandya, D. N. (1981) Insular interconnections with the amygdala in the rhesus monkey. Neuroscience 6: $1231-1248$.

Mufson, E. J. and Mesulam, M.-M. (1982) Insula of the old world monkey. II. Afferent cortical input and comments on the claustrum. J. Comp. Neurol. 212: 23-37.

Norgren, R. (1984) Central neural mechanisms of taste. In: Darien-Smith, I. (ed.; Section Editors, Brookhart, J. and Mountcastle, V. B.) Handbook of Physiology-The Nervous System III, Sensory Processes 1 pp. 1087 1128. American Physiological Society, Washington, DC.

Pritchard, T. C., Hamilton, R. B., Morse, J. R., and Norgren, R. (1986) Projections of thalamic gustatory and lingual areas in the monkey, Macaca fascicularis. J. Comp. Neurol. 244: 213-228.

Rolls, B. J., Rolls, E. T., and Rowe, E. A. (1981a) Sensory-specific satiety in man. Physiology and Behavior 27: 137-142.

Rolls, B. J., Rowe, E. A., Rolls, E. T., Kingston, B., and Megson, A. (1981b) Variety in a meal enhances food intake in man. Physiology and Behavior 26: 215-221.

Rolls, B. J., Rowe, E. A., and Rolls, E. T. (1982) How sensory properties of foods affect human feeding behavior. Physiol. Behav. 29: 409-417.

Rolls, B. J., Van Duijenvoorde, P. M., and Rolls, E. T. (1984) Pleasantness changes and food intake in a varied four course meal. Appetite 5: 337 348.

Rolls, E. T. (1975) The Brain and Reward. Pergamon, Oxford.

Rolls, E. T. (1981) Central nervous mechanisms related to feeding and appetite. British Medical Bulletin 37: 131-134. 
60 Taste in macaque monkey

Rolls, E. T., Scott, T. R., Sienkiewicz, Z. J., and Yaxley, S. (1988) The B. G. (eds.) The Neural Basis of Feeding and Reward. Haer Institute for Electrophysiological Research, Brunswick, Maine.

Rolls, E. T. (1986) Neuronal activity related to the control of feeding. In: Ritter, R. C., Ritter, S., and Barnes, C. D. (eds.) Feeding Behavior: Neural and Humoral Controls ch. 6 pp. 163-190. Academic Press, New York.

Rolls, E. T. (1987) Information representation, processing and storage in the brain: analysis at the single neuron level. In: Changeux, J.-P. and Konishi, M. (eds.) The Neural and Molecular Bases of Learning pp. 503-540. Wiley, Chichester

Rolls, E. T. (1988) Functions of neuronal networks in the hippocampus and neocortex in memory. In: Byrne, J. H. and Berry, W. O. (eds.) Neural Models of Plasticity: Theoretical and Empirical Approaches. Academic Press, New York.

Rolls, E. T., Burton, M. J., and Mora, F. (1976) Hypothalamic neuronal responses associated with the sight of food. Brain Res. 111: 53-66.

Rolls, E. T. and Rolls, B. J. (1977) Activity of neurons in sensory, hypothalamic and motor areas during feeding in the monkey. In: Katsuki, Y., Sato, M., Takagi, S., and Oomura, Y. (eds.) Food Intake and Chemical Senses pp. 525-549. University of Tokyo Press, Tokyo.

Rolls, E. T., Judge, S. J., and Sanghera, M. K. (1977) Activity of neurons in the inferotemporal cortex of the alert monkey. Brain Res. 130: 229238.

Rolls, E. T., Sanghera, M. K., and Roper-Hall, A. (1979) The latency of activation of neurons in the lateral hypothalamus and substantia innominata during feeding in the monkey. Brain Res. 164: $121 \quad 135$

Rolls, E. T., Burton, M. J., and Mora, F. (1980) Neurophysiological analysis of brain-stimulation reward in the monkey. Brain Res. 194: 339-357.

Rolls, E. T. and Rolls, B. J. (1982) Brain mechanisms involved in feeding. In: Barker, L. M. (ed.) Psychobiology of Human Food Selection ch. 3 pp. 33-62. AVI Publishing Company, Westport, Connecticut.

Rolls, E. T., Rolls, B. J., and Rowe, E. A. (1983) Sensory-specific and motivation-specific satiety for the sight and taste of food and water in man. Physiol. Behav, 30: 185-192.
Rolls, E. T., Murzi, E., Yaxley, S., Thorpe, S. J., and Simpson, S. J. (1986) Sensory-specific satiety: food-specific reduction in responsiveness of ventral forebrain neurons after feeding in the monkey. Brain Res. 368: 79-86.

Rolls, E. T., Scott, T. R., Sienkiewicz, Z. J., and Yaxley, S. (1988) The responsiveness of neurones in the frontal opercular gustatory cortex of the macaque monkey is independent of hunger. J. Physiol. 397: 1-12.

Sanghera, M. K., Rolls, E. T. and Roper-Hall, A. (1979) Visual responses of neurons in the dorsolateral amygdala of the alert monkey. Exp. Neurol. 63: 610-626.

Sanides, F. (1968) The architecture of the cortical taste nerve areas in squirrel monkey (Saimiri sciureus) and their relationships to insular, sensorimotor and prefrontal regions. Brain Res. 8: 97-124.

Scott, T. R., Yaxley, S., Sienkiewicz, Z. J., and Rolls, E. T. (1986a) Taste responses in the nucleus tractus solitarius of the behaving monkey. J. Neurophysiol. 55: 182-200.

Scott, T. R., Yaxley, S., Sienkiewicz, Z. J., and Rolls, E. T. (1986b) Gustatory responses in the frontal opercular cortex of the alert cynomolgus monkey. J. Neurophysiol. 56: 876-890.

Sudakov, K., Maclean, P. D., Reeves, A., and Marini, R. (1971) Unit study of exteroceptive inputs to claustrocortex in awake, sitting squirrel monkeys. Brain Res. 28: 19-34

Wiggins, L. L., Rolls, E. T., and Baylis, G. C. (1987) Afferent connections of the caudolateral orbitofrontal cortex taste area of the primate. Chemical Senses 12: 206.

Yaxley, S., Rolls, E. T., Sienkiewicz, Z. J., and Scott, T. R. (1985) Satiety does not affect gustatory activity in the nucleus of the solitary tract of the alert monkey. Brain Res. 347: 85-93.

Yaxley, S., Rolls, E. T., and Sienkiewicz, Z. J. (1989) Gustatory responses of single neurons in the insula of the macaque monkey. J. Neurophysiol. (in press).

Yaxley, S., Rolls, E. T., and Sienkiewicz, Z. J. (1988) The responsiveness of neurons in the insular gustatory cortex of the macaque monkey is independent of hunger. Physiology and Behavior 42: 223-229. 